HU-SEFT R 1994-06

\title{
Conserved Quantities and Electroweak Phase Transitions
}

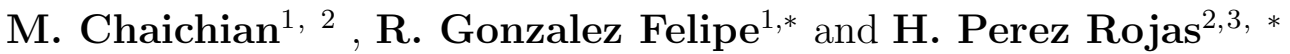

\begin{abstract}
Some cosmological consequences of including the adequate conserved quantities in the density matrix of the electroweak theory are investigated. Several arguments against including the charges associated to the spontaneously broken symmetry are presented. Special attention is focused on the phenomenon of $W$-boson condensation and its interplay with the phase transition for the symmetry restoration is considered. The emerging cosmological implications, such as on the baryon and lepton number densities, are of interest.
\end{abstract}

\footnotetext{
${ }^{1}$ Laboratory of High Energy Physics, Department of Physics, P.O. Box 9 (Siltavuorenpenger 20C) FIN-00014, University of Helsinki, Finland

${ }^{2}$ Research Institute for High Energy Physics, P.O. Box 9 (Siltavuorenpenger 20C) FIN-00014, University of Helsinki, Finland

${ }^{3}$ Research Institute for Theoretical Physics, P.O. Box 9 (Siltavuorenpenger 20C) FIN-00014, University of Helsinki, Finland

* On leave of absence from Grupo de Física Teórica, ICIMAF, Academia de Ciencias de Cuba, Calle E No. 309,Vedado, La Habana 4, Cuba
} 
After the suggestion made by Kirzhnitz and Linde [1] about the mechanism of symmetry restoration by temperature, electroweak phase transitions became a subject of continuous interest (see, e.g. [2]). Among other reasons, this interest has increased in the past few years after the observation that nonperturbative processes (mediated by sphalerons) are relevant to the problem of the baryon asymmetry of the Universe [3, 4].

In this letter we address some specific features which are essential in the formulation of the basic statistical theory underlying the thermodynamics of the standard electroweak model, in which the notions of gauge invariance, spontaneously broken symmetries and Higgs mechanism play a basic role. We shall consider the consequences of including the adequate conserved charges, together with the Hamiltonian, in the density matrix. When studying the electroweak phase transitions the canonical ensemble is the one usually used. The latter, however, restricts the domain of physical applications, especially as one would like to incorporate the baryon asymmetry of the Universe and the equilibrium among electrically charged particles. In some previous papers [5, 6], it has been pointed out that the grand canonical ensemble must be used. Moreover, as emphasized in [7], the conserved quantities to be included must be essentially the electric charge and the lepton and baryon numbers. Thus, the weak neutral charge associated with the spontaneously broken symmetry must be excluded.

Here we would like to discuss the above-mentioned points in connection with a new phase transition which arises when these charges are included, namely the $W$-vector boson condensation (which for high temperatures occurs at very low lepton densities) [5, 7] and its interplay with the phase transition of symmetry restoration, which according to the recent results [8] is of first order if the perturbative expansion is taken beyond the one-loop approximation.

The basic idea in [1] came out from the analogy between the Weinberg-Salam Lagrangian and the Ginzburg-Landau free energy for superconductivity which is regarded as a condensate of Cooper pairs. In the electroweak case, the role of the superconductor ground state is played by the vacuum whose symmetry has been spontaneously broken. This breaking of the symmetry is then understood in a hot Universe as a consequence of a phase transition by cooling below some critical temperature $T_{c}$.

One could be tempted to interprete the asymmetric vacuum of electroweak theory as a weak-neutrally charged condensate of scalar particles. However, this picture is not correct once it mixes the concepts of statistics (many-particle systems) with those of quantum field theory. By relativistic invariance we must require that for the vacuum $P_{\mu}|0\rangle=0$, i.e. the four-momentum vector must have zero eigenvalue. Obviously, a condensate of scalar particles would violate this condition.

In a correct interpretation, however, the vacuum is asymmetric at any temperature and the symmetry breakdown is characterized by some parameter $\xi=a$, which is the minimum of an effective potential in quantum field theory. By heating, the effective field amplitude (related to the nonzero vacuum charge, see below) is decreased to the value $\xi^{2}(T)=a^{2}-\alpha T^{2}$ due to screening, entropy-increasing processes, coming from the temperature quantum loop corrections . At the critical temperature $T_{c}$ we obtain $\xi^{2}(T)=0$ as the solution corresponding to a minimum of the effective temperaturedependent potential. However, this minimum should not be understood as a new vacuum, but as a symmetric ground state $|\psi\rangle$ of a many-particle system. 
Two problems closely related to the above are the questions about the infrared stability of the system (if long-range weak forces appear after the symmetry restoration) and the conservation of the weak neutral charge $Q^{N}$. (Both were considered in 11 and the second one, mentioned in [9]). If the neutrality of the system with respect to the weak neutral charge, i.e. $\left\langle Q^{N}\right\rangle=0$, can be defined at all, then it would be reasonable to require it only in the symmetric phase in order to prevent long-range repulsive forces from making the system unstable. This argument apparently becomes weakened if some small infrared mass of order $g^{2} T$ appears [10. Thus it is usually accepted that $\left\langle Q^{N}\right\rangle$ is a conserved quantity only for $T>T_{c}$. One should understand this conservation in a statistical and not quantum mechanical sense, which means that $\operatorname{Tr}\left(\rho \dot{Q}^{N}\right)=\operatorname{Tr}\left(i \rho\left[H, Q^{N}\right]\right)=f(\xi(T), T)$ vanishes for $T>T_{c}$, without the requirement $\left[H, Q^{N}\right]=0$. However, this conservation does not occur below $T_{c}$ and moreover $Q^{N}$ becomes in quantum field theory an ill-defined operator [11, 12]. Indeed, according to Coleman's theorem [13] a charge operator $Q$ annihilates the vacuum, i.e. $Q|0\rangle=0$, is conserved, $[H, Q]=0$. However, a charge associated with a spontaneously broken symmetry like $Q^{N}$ does not annihilate the vacuum, $Q^{N}|0\rangle \neq 0$ [12]. As a consequence, the operator $Q^{N}$ has many odd features: one cannot construct states of definite charge number and even define the properties of conjugation and composition law . Furthermore, it has been stated that such an operator is non-Hermitean [11] and for finite energy conditions, even non-conserved at the classical level [12]. The above arguments forbid the inclusion of $Q^{N}$ into the density matrix via a chemical potential. This can be understood also from the facts that such a charge does not lead to irreducible representations of states with definite charge number in specific Hilbert spaces once it mixes representations [12], and also that it forbids superselection rules.

A fundamental quantum-statistical statement is that equilibrium states satisfy the socalled Kubo-Martin-Schwinger (KMS) condition [14, 15], which requires that the density matrix $\rho$ as well as $H^{\prime}=H-\sum_{i} \mu_{i} N_{i}$ have a discrete spectrum. The KMS condition is obtained from the time translations of any operator $A$ given by $e^{i H^{\prime} t} A e^{-i H^{\prime} t}$, with the transformation which involves the chemical potentials being a $U(1)$ gauge transformation. The introduction of $Q^{N}$ would lead to non-unitary implementable transformations invalidating the KMS condition.

The above arguments, applied to the grand canonical ensemble for the electroweak model, imply that only those charges that belong to the center of the unbroken part of the $S U(2)_{L} \times U(1)$ group satisfy the KMS condition and thus can have a chemical potential [15]. In other words, only baryon and lepton numbers as well as electric charge can be included in the density matrix via their corresponding chemical potentials. It could be argued that in the case of electric charge, the chemical potential added to the fourth component of the charged gauge field can be gauged away. However, since there is only an analogy and not an equivalence between quantum statistics and quantum field theory via the Bloch equation and the Matsubara formalism, this chemical potential is to be understood at most as the analog of a constant background field, not subject to gauge transformations (see e.g. [16]) .

We should emphasize that if one does what one should not do, and includes a chemical potential $\mu_{3}$ corresponding to the spontaneously broken weak neutral charge, then one arrives at drastic changes in the consequences of the electroweak theory applications in cosmology [7]. In particular, if such a chemical potential is introduced [17], one obtains 
a relation between the critical temperature for the symmetry restoration and the lepton density, which in turn relates the latter to the photon density. This then leads to the prediction that the lepton density is by several orders of magnitude larger than the baryon density, a belief which has been accepted starting from the work by Linde [17]. According to our arguments such a chemical potential should not be included and then one does not necessarily obtain the large abundance of lepton over baryon densities. A preference for the latter situation has been advertised [18 from different considerations and revisited cosmological models. In fact, as we shall see below, a lepton density of the same order of magnitude as the baryon density will emerge from the results of the present letter.

Taking into account the previous arguments, let us turn now our attention to the problem of $W$-boson condensation, first pointed out by Linde [19] for zero temperature but at finite densities. In [7] we have discussed the general formalism to be used in order to obtain the thermodynamic quantities. The effective potential $V(\xi)$, essentially off-shell, is adequate for the discussion of the symmetry restoration phase transition, whereas for obtaining the charge densities $N_{i}=-\partial \Omega / \partial \mu_{i}$, the thermodynamic potential $\Omega=V(\xi(T))$ should be used. In [5], [7] the phase diagram for $W$-boson condensation was obtained assuming that the symmetry restoration phase transition was second order. As mentioned above, it has been argued that such transition can become first order if high-order terms in the loop expansion are included. Therefore, it would be of interest to investigate also the consequences of including such terms in the problem of $W$-condensation. Recently, the effective potential of the standard model was obtained beyond the one-loop when perturbative expansions include ring diagrams [20]. The problem of the coefficient of the term linear in the temperature in the one-loop approximation is cured by temperaturedependent radiative corrections and this term essentially decides the nature of the phase transition to be a first order one.

The conditions for condensation are obtained from the infrared poles of the (gaugedependent) $W$-boson Green function

$$
\lim _{k_{4}=0, \vec{k} \rightarrow 0} \operatorname{Det}_{\mu \nu}^{-1}\left(k_{4}-i \mu_{W}, \vec{k}, M_{W}\right)=0,
$$

where $\mu_{W}$ is the chemical potential associated with the electric charge, $M_{W}$ is the $W$ boson mass, $D_{\mu \nu}^{-1}=D_{0 \mu \nu}^{-1}+\Pi_{\mu \nu} ; D_{0 \mu \nu}^{-1}$ is the free propagator and $\Pi_{\mu \nu}$ is the polarization operator. Eq. (1) leads to different conditions for the condensation of transverse and longitudinal modes, the latter acquiring some extra mass due to the Debye screening [20]. We have then

$$
\mu_{W}^{2}=M_{W}^{2}, \quad \mu_{W}^{2}=M_{W}^{2}+g^{2} T^{2}\left(\frac{5}{6}+\frac{n_{f}}{3}\right),
$$

where $g=e / \sin \theta_{W}$ is the $S U(2)$ coupling constant and $n_{f}$ is the number of fermion generations. For simplicity we shall consider in what follows only one generation of leptons and quarks, i.e. $n_{f}=1$. As both equations cannot be satisfied simultaneously, we conclude that the condensation of the transverse modes is to be expected, which leads to the solutions $\mu_{W}= \pm M_{W}$. Which one of them will be realized depends on the sign of the background charge. 
In the one-loop approximation considered in [7], we found equal masses for longitudinal and tranverse modes, which was interpreted as a consequence of the rotational invariance. However, the Debye mass for the longitudinal modes appears already in the QED plasma without destroying the rotational invariance. Instead of transverse and longitudinal modes, it would be better to speak of magnetic and electric modes respectively, the tensor structure of the both preserving the rotational invariance. The condensation of the electrically charged transverse modes has also an interesting analogy with what happens in the QED plasma. If we consider a very hot photon gas in equilibrium with a neutral electron-positron background, we encounter the property that electric fields are screened by the Debye term, but that it can exist in equilibrium in the presence of a constant magnetic field; this is determined by the infrared behavior of the photon Green function. We can understand then the external magnetic field as a sort of condensate of transverse photons. However, in our present case it is the charge and not the field which condensates.

In what follows we take the critical temperature for $W$-boson condensation (of the order of $100 \mathrm{GeV}$ ) to be above the confinement temperature (of the order of $100 \mathrm{MeV}$ ), a situation which is guaranteed when the lepton density is sufficiently high (for a lower bound estimate of the latter one can use Eq. (7)).

The asymptotic equilibrium equations for electric charge, lepton and baryon number conservation read as follows:

$$
\begin{gathered}
\frac{\partial \Omega}{\partial \mu_{W}}=-\frac{2}{3} N_{u}+\frac{1}{3} N_{d}+N_{e}+N_{W}=0 \\
\frac{\partial \Omega}{\partial \mu_{2}}=N_{e}+N_{\nu}=\ell \\
\frac{\partial \Omega}{\partial \mu_{4}}=N_{u}+N_{d}=b
\end{gathered}
$$

where $\mu_{2}$ and $\mu_{4}$ correspond to lepton and baryon number conservation respectively and are introduced into the density matrix as factors multiplying the corresponding conserved Noether charges. In $(3), N_{i}, i=u, d, e, W, \nu$, denote the net number density of the corresponding particles minus their antiparticles.

Asymptotically we have for fermions

$$
N_{f L}=N_{f R}=\frac{\mu_{f} T^{2}}{6}
$$

while for $W$-bosons

$$
N_{W}=\left(1-\frac{g}{\pi} \sqrt{\frac{7}{6}}\right) \mu_{W} T^{2} .
$$

From the interaction vertices we also have in the equilibrium the following relations:

$$
\mu_{e}=\mu_{W}+\mu_{2}, \mu_{u}=-2 \mu_{W} / 3+\mu_{4}, \mu_{d}=\mu_{W} / 3+\mu_{4} .
$$

By solving Eqs. (3) and taking the condition of condensation for the transverse modes as $\mu_{W}=-M_{W}=-g \xi(T) / 2$ (i.e. taking the $\mu_{W}=-M_{W}$ solution in order to obtain 
the situation $\ell-b / 4>0$ ), we obtain finally the critical curve for the condensation of transverse modes

$$
\ell-\frac{b}{4}=-\frac{23}{12} \mu_{W} T^{2}=\frac{23}{24} g T^{2} \xi(T)
$$

where the expression for $\xi(T)$ is obtained from the minimum of the effective potential. If the phase transition is of second order, $\xi(T)$ decreases smoothly with increasing temperature, and the phase diagram has qualitatively the same form as the one obtained in [7]. The more interesting case results when the nature of the phase transition is first order . Then in the process of cooling $\xi(T)$ jumps abruptly from zero to a positive value with the formation of bubbles inside which $\xi(T) \neq 0$. The phase diagram is depicted in Fig. 1 .

We must point out here that the condensation of $W$-bosons partially diminishes the $m^{3} T$ term needed for the first order phase transition to take place. This is due to the fact that the $W$-bosons contribute to the effective potential with a term of the form $\left(M_{W}^{2}-\mu_{W}^{2}\right)^{3 / 2} T /(6 \pi)$, which vanishes if the condensation arises. We believe, however, that the contributions of the longitudinal and Higgs modes are enough to keep the linear in $T$ term necessary for a first order phase transition.

Now if we assume that the effective density $\ell-b / 4$ in (7) is large enough to preserve the condensate after the symmetry breaking, it is easy to understand that the relative abundance of isotopic components of leptons and quarks are strongly changed by the phase transition. Indeed, we have the chemical equilibrium equations

$$
\mu_{u}-\mu_{d}=\mu_{\nu}-\mu_{e}=-\mu_{W}=M_{W} .
$$

According to Eqs. (8), in the symmetric phase $\left(M_{W}=0\right)$ there exists equal relative abundance of $u$ and $d$ quarks, as well as of electrons and neutrinos. After the breaking of the symmetry, the $W$-boson condensation which is induced by the lepton density leads to a displacement of the equilibrium with a resulting average excess of $u$ over $d$ quarks. This could favour the formation of an excess of protons over neutrons with the cooling of the Universe below the confinement critical temperature (which corresponds to the present state of the Universe), if the processes which dominate at lower temperatures, such as nucleosynthesis, do not totally erase this asymmetry. We notice that identical to (8) equations are separately valid for other lepton and quark generations and thus similar conclusions can be drawn for them as well.

There might also exist a connection between the $W$-boson condensation mechanism and a partial understanding of the baryon asymmetry of the Universe, first treated by Sakharov in his pioneer work [21] using a microscopic as well as a thermodynamic approach. In the broken phase, according to Eqs. (3) and (4), one would have $\mu_{u}+\mu_{d}=$ $3 b / T^{2}$. With the occurrence of $W$-condensation, according to Eq. (8) one could expect a value of order $M_{W} T^{2} / 3$ for $b$, leading to an enhancement of the baryon number density. We would like to mention that similarly, from Eqs. (3) and (4), it follows that the lepton number density $\ell$ is also expected to have a value of the same order of magnitude as the baryon number density $b$, in contrast to the result $\ell \gg b$ obtained in [17] in an equivalent way if the spontaneously broken weak neutral charge would have been taken into account as a conserved quantity. The equality, in the order of magnitude, of $\ell$ and $b$ 
has been argued for 18 from entirely different cosmological considerations. We thus consider that the phenomenon of $W$-boson condensation may have interesting cosmological implications.

\section{Acknowledgements}

We are grateful to J. Ignatius, K. Kajantie, C. Montonen, V. Semikoz and M. Shaposhnikov for useful discussions and comments.

\section{Figure captions}

Fig. 1 Phase diagram for the high temperature $W$-condensation in the case of first-order phase transition. 


\section{References}

[1] D.A. Kirzhnitz and A.D. Linde, Phys. Lett. 42B (1972) 471; Ann. Phys. 101 (1976) 195.

[2] J. R. Espinosa, M. Quiros and F. Zwirner, Phys. Lett. B 307 (1993) 106.

[3] V. A. Kuzmin, V. A. Rubakov and M. Shaposhnikov, Phys. Lett. 155B (1985) 36.

[4] G. F. Giudice and M. Shaposhnikov, Phys. Lett. B 326 (1994) 118.

[5] H.Perez Rojas and O.K.Kalashnikov, Nucl. Phys. B293 (1987) 241.

[6] E.J.Ferrer, V.de la Incera and A.E.Shabad, Phys. Lett. 185B (1987) 407; Nucl. Phys. B309 (1988) 120.

[7] M. Chaichian, R. Gonzalez Felipe, D. Louis Martinez and H. Perez Rojas, Z. Phys. C (to appear, 1994).

[8] G. W. Anderson and L. J. Hall, Phys. Rev. D 45 (1992) 2685; M. Dine, R. G. Leigh, P. Huet, A. Linde and D. Linde, Phys. Rev. D 46 (1992) 550; B.-H. Liu, L. McLerran and N. Turok, Phys. Rev. D 46 (1992) 2668; M. E. Carrington and J.I. Kapusta, Phys. Rev. D 47 (1993) 5304.

[9] S.Weinberg, Phys. Rev. D 9 (1974) 3357.

[10] A. M. Polyakov, Phys. Lett. 72B (1978) 477.

[11] E.Gal-Ezer and H.Reeh, Commun. Math. Phys. 43 (1975) 137.

[12] M. Chaichian, C. Montonen and H. Perez Rojas, Phys. Lett. B 256 (1991) 227; M. Chaichian, J. Gonzalez, C. Montonen and H. Perez Rojas, Phys. Lett. B 300 (1993) 118.

[13] S.Coleman, J. Math. Phys. 7 (1966) 787.

[14] H. Araki, D. Kastler,M. Takesaki and R. Haag, Commun. Math. Phys. 53 (1977) 97.

[15] R. Haag, Local Quantum Physics (Springer, Berlin, 1992).

[16] D. G. Boulware, Phys. Rev. D 23 (1981) 389; B. S. DeWitt, Quantum Gravity II (Oxford Univ. Press, 1981).

[17] A. D. Linde, Rep. Prog. Phys. 42 (1979) 389.

[18] K. A. Olive, D. N. Schramm, D. Thomas and T. P. Walker, Phys. Lett. B 265 (1991) 239.

[19] A. D. Linde, Phys. Lett. 86B (1979) 39.

[20] Z. Fodor and A. Hebecker, preprint DESY-94-025 (1994).

[21] A. D. Sakharov, JETP Lett. 5 (1967) 24. 
This figure "fig1-1.png" is available in "png" format from: http://arxiv.org/ps/hep-th/9405170v1 